\title{
Dynamic model atmospheres of AGB stars
}

\section{Effects of frequency-dependent radiative transfer}

\author{
S. Höfner ${ }^{1}$, R. Gautschy-Loidl ${ }^{2}$, B. Aringer ${ }^{3}$, and U. G. Jørgensen ${ }^{3}$ \\ 1 Dept. of Astronomy and Space Physics, Uppsala University, Box 515, 75120 Uppsala, Sweden \\ 2 Rüti, Switzerland \\ e-mail: rita@gautschy.ch \\ 3 Niels Bohr Institute, Astronomical Observatory, Juliane Maries Vej 30, 2100 Copenhagen, Denmark
}

Received 26 June 2002 / Accepted 26 November 2002

\begin{abstract}
We present a new generation of model atmospheres for AGB stars which combine time-dependent dynamics and frequency-dependent radiative transfer. This allows us to take both the effects of pulsation (shock waves, stellar winds) and the complex influence of molecular opacities into account. In the case of C-rich stars, the models also include a self-consistent time-dependent description of dust formation. We investigate the influence of frequency-dependent radiative transfer on the energy and momentum balance of the atmosphere and compare our new models to existing grey dynamical models as well as to classical hydrostatic model atmospheres. We stress the importance of non-grey radiative transfer for obtaining realistic density-temperature structures even in highly dynamical models, discussing both the resulting observable properties and the wind characteristics. Presenting synthetic spectra, we argue that the current dynamical models represent an important step in a process leading from a qualitative to a quantitative description of atmospheres and winds of pulsating AGB stars.
\end{abstract}

Key words. stars: AGB and post-AGB - stars: variables: general - stars: atmospheres - stars: mass-loss - hydrodynamics radiative transfer

\section{Introduction}

Atmospheres of cool pulsating Asymptotic Giant Branch stars present a major challenge for realistic, self-consistent modelling: Propagating shock waves caused by stellar pulsation modify the structure of the atmosphere on local and global scales, causing strong deviations from hydrostatic stratification. The radiative field is dominated by molecular opacities or even by dust grains forming in the cool outer layers of the atmospheres. Important micro-physical processes like chemistry and dust formation may be severely out of equilibrium.

Given the performance of existing computers it is not feasible at present to model the coupled system of time-dependent hydrodynamics, detailed non-LTE radiative transfer and chemical non-equilibrium. In particular, it would not be practicable to use such models for studying a reasonable number of stars with different parameters. Therefore it is important to identify the most relevant processes in order to create models which are sufficiently realistic to allow a reliable interpretation of current observations but still efficient enough from a computational point of view to be applicable for more than a few hand-picked objects.

Send offprint requests to: S. Höfner, e-mail: hoefner@astro.uu.se
At present, two types of models exist side by side. Classical hydrostatic model atmospheres (e.g. Alvarez et al. 2002; Jørgensen et al. 2000; Loidl et al. 2001) include a sophisticated treatment of micro-physical processes and radiative transfer but neglect the effects of dynamics due to pulsation and winds. On the other hand, time-dependent dynamical models for stellar winds (e.g. Höfner \& Dorfi 1997; Winters et al. 2000) tend to be based on crude descriptions of radiative transfer and simplified micro-physics, leading to unrealistic atmospheric densitytemperature structures.

Our recently developed model atmospheres which couple time-dependent dynamics and frequency-dependent radiative transfer try to bridge this gap. A first prototype of these models was presented by Höfner (1999). We have further improved these models by including a non-grey description of the dust component and have discussed a few selected models with different parameters (e.g. Höfner et al. 2002). Preliminary studies of observable properties calculated from these new dynamical models (spectra at various resolutions and their temporal variations) show drastic improvements compared to the synthetic spectra based on previous (grey) generations of models (e.g. Aringer et al. 2002a; Loidl et al. 2000).

The models discussed here are primarily intended for AGB stars with effective temperatures lower than about $3500 \mathrm{~K}$ which undergo large-amplitude radial pulsations (Mira and SR 
variables), with and without dust-driven stellar winds. The spectra and colours of hotter AGB stars with low pulsation amplitudes can often be described reasonably well with classical hydrostatic model atmospheres (e.g. Alvarez \& Plez 1998; Jørgensen et al. 2000; Loidl et al. 2001; Aringer et al. 2002b) which require a smaller computational effort. Existing grey dynamical models in the literature usually specialise on heavily dust-enshrouded objects (e.g. IR carbon stars; Winters et al. 1997). In contrast, our new generation of models has also been developed with the goal of predicting spectra of stars with low mass loss rates and circumstellar envelopes of moderate optical depths which simultaneously show spectral features originating in photospheric layers and the stellar wind. This may be an important step to improve the understanding of mass loss mechanisms in AGB stars by detailed comparisons of models and observations.

In this paper we present the physical input and general results of our new frequency-dependent dynamical models. We discuss the effects of non-grey radiative transfer on the structure of the atmospheres and on the formation of dust-driven winds. The non-grey models are compared to models based on Planck-mean opacities presented in the preceding papers in this series (Höfner et al. 1998; Loidl et al. 1999). We have performed a set of calculations where we have systematically varied the stellar parameters. The effects on the resulting synthetic spectra will be discussed in detail in a forthcoming paper. Here, we restrict the discussion of observable properties to a short overview of the progress and remaining problems with this new generation of dynamical model atmospheres.

\section{Modelling method}

The models cover a spherical shell with an inner boundary below the stellar photosphere but above the driving zone of the pulsation. The effects of stellar pulsation are simulated by temporal variations at the inner boundary. The location of the outer boundary depends on the dynamics of the model under consideration: for models which form stellar winds it is typically at a distance of 20-30 stellar radii (allowing outflow), for models without a wind the outer boundary is close to the photosphere, following the movements of the upper atmospheric layers.

The variable structure of the atmosphere is described by the equations of hydrodynamics (equation of continuity, equation of motion and energy equation) together with the frequencyintegrated zeroth and first moment equations of the radiative transfer equation (accounting for the energy and momentum balance of the radiative field). In the case of C-rich stars, a set of so-called moment equations describing the time-dependent formation, growth and evaporation of dust grains is solved simultaneously with the radiation-hydrodynamical equations.

\subsection{Basic equations: Frequency-dependent vs. grey}

The grey version of these equations has been discussed in previous papers (see Höfner et al. 1995 and references therein). To make the physical differences between our previous grey models and the new generation of frequency-dependent dynamical
Table 1. List of symbols.

\begin{tabular}{cl}
\hline \hline$r$ & radius coordinate \\
$t$ & time \\
$m_{\mathrm{r}}$ & mass within radius $r$ \\
$\rho$ & gas density \\
$e$ & specific internal gas energy \\
$u$ & matter velocity \\
$J$ & zeroth moment of the radiation intensity \\
$H$ & first moment of the radiation intensity \\
$K$ & second moment of the radiation intensity \\
$K_{j}$ & moments of the grain size distribution \\
& \\
$P_{\mathrm{g}}$ & gas pressure \\
$T_{\mathrm{g}}$ & gas temperature \\
$T_{\mathrm{r}}$ & radiation temperature \\
$T_{\mathrm{d}}$ & dust grain temperature \\
$S_{\mathrm{g}}$ & source function of the gas \\
$\kappa_{v}$ & mass absorption coefficient of the gas \\
$\chi_{v}$ & absorption coefficient of the dust \\
$1 / \tau$ & net growth-rate of the dust grains \\
$\mathcal{J}$ & net grain formation rate per volume \\
$N_{\ell}$ & lower size limit of grains contributing to $K_{j}$ \\
& \\
$c$ & speed of light \\
$G$ & constant of gravitation \\
$\sigma$ & Stefan-Boltzmann constant \\
\hline &
\end{tabular}

atmospheres presented here more clear we give an overview of the modified set of equations.

\subsubsection{Radiation hydrodynamics}

The gas component is described by the three conservation laws for mass, momentum and energy,

$\frac{\partial}{\partial t} \rho+\nabla \cdot(\rho u)=0$

$\frac{\partial}{\partial t}(\rho u)+\nabla \cdot(\rho u u)=-\nabla P_{\mathrm{g}}-\frac{G m_{r}}{r^{2}} \rho+\frac{4 \pi}{c}\left(\kappa_{\mathrm{H}} \rho+\chi_{\mathrm{H}}\right) H$

$\frac{\partial}{\partial t}(\rho e)+\nabla \cdot(\rho e u)=-P_{\mathrm{g}} \nabla \cdot u+4 \pi \rho\left(\kappa_{\mathrm{J}} J-\kappa_{\mathrm{S}} S_{\mathrm{g}}\right)$

(see Table 1 for an explanation of the symbols). The integrated mass inside a radius $r$ is defined as

$m_{r}=\int_{0}^{r} 4 \pi r^{\prime 2} \rho \mathrm{d} r^{\prime}$

The source terms describing the net energy and momentum exchange between the gas and the radiation field are frequency integrals, i.e.

$$
\begin{aligned}
& \kappa_{\mathrm{J}} J-\kappa_{\mathrm{S}} S_{\mathrm{g}}=\int_{0}^{\infty} \kappa_{v}\left(J_{v}-S_{v}^{\text {gas }}\right) \mathrm{d} v \\
& \kappa_{\mathrm{H}} H=\int_{0}^{\infty} \kappa_{v} H_{v} \mathrm{~d} v \\
& \chi_{\mathrm{H}} H=\int_{0}^{\infty} \chi_{v} H_{v} \mathrm{~d} v
\end{aligned}
$$


where the frequency-integrated opacities are defined by

$\kappa_{\mathrm{X}}=\frac{\int_{v} \kappa_{v} X_{v} \mathrm{~d} v}{X} \quad$ and $\quad \chi_{\mathrm{X}}=\frac{\int_{v} \chi_{\nu} X_{v} \mathrm{~d} v}{X}$.

Here $X$ corresponds to one of the quantities $J, H$ or $S$, and variables without the subscript $v$ denote the frequency-integrated values $X=\int_{v} X_{v} \mathrm{~d} v$.

The frequency-integrated moments of the radiation intensity $J$ and $H$ are determined by the zeroth and first order moment equations of the radiative transfer equation

$$
\begin{aligned}
\frac{1}{c} \frac{\partial}{\partial t} J+\frac{1}{c} \nabla \cdot(J u)= & -\nabla \cdot H-\frac{1}{c} K \nabla \cdot u+\frac{u}{c} \frac{3 K-J}{r} \\
& -\rho\left(\kappa_{\mathrm{J}} J-\kappa_{\mathrm{S}} S_{\mathrm{g}}\right) \\
\frac{1}{c} \frac{\partial}{\partial t} H+\frac{1}{c} \nabla \cdot(H u)= & -\nabla K-\frac{3 K-J}{r}-\frac{1}{c} H \nabla u \\
& -\left(\kappa_{\mathrm{H}} \rho+\chi_{\mathrm{H}}\right) H .
\end{aligned}
$$

As long as velocities are small compared to the speed of light (which is the case in AGB star atmospheres) the terms of order $u / c$ in these equations are negligible and the equations take the more familiar form

$$
\begin{aligned}
& \nabla \cdot H+\rho\left(\kappa_{\mathrm{J}} J-\kappa_{\mathrm{S}} S_{\mathrm{g}}\right)=0 \\
& \nabla K+\frac{3 K-J}{r}+\left(\kappa_{\mathrm{H}} \rho+\chi_{\mathrm{H}}\right) H=0 .
\end{aligned}
$$

Note, however, that our code contains the more general form as given in Eqs. (9)-(10) since the radiation-hydrodynamical part has been developed for a wider application.

To calculate the opacities $\kappa_{\mathrm{J}}, \kappa_{\mathrm{H}}$ and $\kappa_{\mathrm{S}}$ (and the corresponding values for the dust opacity $\chi$ ) which are required in the radiation-hydrodynamical equations listed above the frequency-depended moments of the radiative intensity $J_{v}$ and $H_{v}$ have to be known. These moments as well as the Eddington factor $f_{\text {edd }}=K / J$ which is needed to close the system of moment equations Eqs. (9)-(10) are obtained by solving the frequency-dependent equation of radiative transfer for a given density-temperature structure at each time-step using the method of characteristics. The models presented here are based on opacity sampling data of molecular opacities (SCAN data base, Jørgensen 1997) for $\mathrm{CO}, \mathrm{CH}, \mathrm{C}_{2}, \mathrm{CN}, \mathrm{C}_{2} \mathrm{H}_{2}, \mathrm{HCN}$, $\mathrm{C}_{3}, \mathrm{TiO}, \mathrm{H}_{2} \mathrm{O}$ and $\mathrm{SiO}$. The radiative transfer equation is solved at 51 frequency points between 0.25 and $12.5 \mu \mathrm{m}$. These frequency points are roughly equidistant in wavenumber (and therefore in energy) but randomly distributed relative to opacity features as required by a statistical approach (opacity sampling). Assuming LTE, the source function is given by

$S_{v}=\frac{\rho \kappa_{v} B_{v}\left(T_{\mathrm{g}}\right)+\chi_{v} B_{v}\left(T_{\mathrm{d}}\right)}{\rho \kappa_{v}+\chi_{v}}$

where $B_{v}(T)$ denotes the Planck function for a temperature $T$.

The system of hydrodynamical equations requires a closing condition in the form of an equation of state for the gas, specifying the thermal pressure $P_{\mathrm{g}}$ and the temperature $T_{\mathrm{g}}$ as a function of the primary variables $\rho$ and $e$. To keep the models comparable to previous ones and to isolate the influence of non-grey radiative transfer we use the same assumptions about the equation of state as in earlier papers, i.e. a perfect gas with $\gamma=5 / 3$ and $\mu=1.26$.

\subsubsection{Dust formation}

The C-rich models presented here include a time-dependent description of dust grain growth and evaporation using the moment method (Gail \& Sedlmayr 1988; Gauger et al. 1990). In this approach, the dust component is described in terms of moments $K_{j}$ of the grain size distribution function weighted with a power $j$ of the grain radius. The moment $K_{0}$ represents the total number density of grains (integral of the size distribution function over all grain sizes) while $K_{3} / K_{0}$ is proportional to the average volume of the grains. We solve the equations which determine the evolution of the dust component

$\frac{\partial}{\partial t} K_{0}+\nabla \cdot\left(K_{0} u\right)=\mathcal{J}$
$\frac{\partial}{\partial t} K_{j}+\nabla \cdot\left(K_{j} u\right)=\frac{j}{3} \frac{1}{\tau} K_{j-1}+N_{\ell}^{j / 3} \mathcal{J}$

$(1 \leq j \leq 3)$ together with the radiation-hydrodynamics system described above, assuming spherical grains consisting of amorphous carbon (see Table 1 for the definition of symbols). The nucleation, growth and evaporation of grains is assumed to proceed by reactions involving $\mathrm{C}, \mathrm{C}_{2}, \mathrm{C}_{2} \mathrm{H}$ and $\mathrm{C}_{2} \mathrm{H}_{2}$.

The frequency-dependent opacity of dust grains can be expressed in terms of the extinction efficiency $Q_{\text {ext }}$ (the ratio of the extinction cross section to the geometrical cross section of the grain). As the grains are small compared to the wavelengths where most of the flux is emitted, the opacities can be treated in the small particle limit for the frequency integrals defined above. In this case the quantity $Q_{\text {ext }}^{\prime}=Q_{\text {ext }} / a_{\text {gr }}$ becomes independent of the grain radius $a_{\mathrm{gr}}$ and the dependence of the opacity on wavelength and grain size can be separated into two independent factors. Therefore, the absorption coefficient can be written as

$$
\begin{aligned}
\chi_{\lambda} & =\int_{0}^{\infty} a_{\mathrm{gr}}^{2} \pi Q_{\mathrm{ext}}\left(a_{\mathrm{gr}}, \lambda\right) n\left(a_{\mathrm{gr}}\right) \mathrm{d} a_{\mathrm{gr}} \\
& =Q_{\mathrm{ext}}^{\prime}(\lambda) \pi \int_{0}^{\infty} a_{\mathrm{gr}}^{3} n\left(a_{\mathrm{gr}}\right) \mathrm{d} a_{\mathrm{gr}}
\end{aligned}
$$

where $n\left(a_{\mathrm{gr}}\right) \mathrm{d} a_{\mathrm{gr}}$ is the number density of grains in the grain radius interval between $a_{\mathrm{gr}}$ and $a_{\mathrm{gr}}+\mathrm{d} a_{\mathrm{gr}}$. The last integral is proportional to the moment $K_{3}$, given by the equations above.

The models in this paper are calculated using the data of Rouleau \& Martin (1991) for $Q_{\text {ext }}^{\prime}$ (see Fig. 6). Apart from the opacity, we keep the same microphysical grain properties and chemical assumptions as in our previous papers for reasons of comparability (see Höfner et al. 1995 and references therein). Here, we are mainly interested in a direct comparison of our new models with existing grey ones. However, to obtain realistic results (including observable properties), a consistent choice of microphysical properties and opacities for dust grains is crucial as recently demonstrated by Andersen et al. (2002).

The presence of dust grains influences both the energy and momentum balance of the atmosphere. For simplicity and comparability with previous models we assume complete momentum coupling of gas and dust, i.e. the momentum gained by the dust from the radiation field is directly transferred to the gas (last term in Eq. (2)). On the other hand, the transfer of internal energy between gas and dust is negligible compared 
to the interaction of each component with the radiative field (cf. Gauger et al. 1990). Therefore we assume that the grain temperature is given by the condition of radiative equilibrium

$\chi_{\mathrm{J}} J-\chi_{\mathrm{S}} S\left(T_{\mathrm{d}}\right)=0 \longrightarrow T_{\mathrm{d}}=\left(\frac{\chi_{\mathrm{J}}}{\chi_{\mathrm{S}}}\right)^{1 / 4} T_{\mathrm{r}}$

where $S\left(T_{\mathrm{d}}\right)=B\left(T_{\mathrm{d}}\right)=T_{\mathrm{d}}^{4} \sigma / \pi$ and the radiation temperature is defined as

$T_{\mathrm{r}}=(J \pi / \sigma)^{1 / 4}$.

At present, our model contains no description of grain formation for an O-rich chemistry. Therefore, when studying $\mathrm{M}$ stars we currently either assume that no dust is present, or use a simple parameterised description of the dust opacity

$\kappa_{\mathrm{d}}^{\mathrm{B} 88}=\frac{\kappa_{\mathrm{d}}^{\max }}{1+\exp \left[\left(T_{\mathrm{r}}-T_{\text {cond }}\right) / \delta\right]}$

as in Bowen (1988) where $\kappa_{\mathrm{d}}^{\max }$ is the maximum value of the dust mass absorption coefficient, $T_{\text {cond }}$ is the condensation temperature and $\delta$ is the width of the step function in temperature. Assuming this simple formula for the dust opacity enables us to explore the qualitative influence of a wind on the atmosphere even for the O-rich case. However, we are aware of the shortcomings of this approach and make no quantitative claims concerning the mass loss of these objects. The Orich models presented here which use Bowen's simple prescription for the dust opacity are included for comparison only. We set $T_{\text {cond }}=1500 \mathrm{~K}$ and $\delta=60 \mathrm{~K}$ but we use different values for $\kappa_{\mathrm{d}}^{\max }$ as specified in Table 3 .

\subsection{Numerical method}

The conservation laws Eqs. (1)-(3), Eqs. (9)-(10) and Eqs. (14)-(15) form a non-linear system of PDEs which is solved implicitly using a Newton-Raphson scheme. The main features of the numerical technique used in our model calculations are the following: a conservative (volume-integrated) formulation of the discretized radiation-hydrodynamics and dust equations, a monotonic second-order advection scheme (van Leer 1977), as well as an adaptive grid. A so-called grid equation is solved simultaneously with the physical conservation equations and distributes the grid points according to accuracy considerations (Dorfi \& Drury 1987). The calculations presented here use 100 radial grid points and the desired resolution is defined by $\rho$ and $e$. Details of the numerical method are discussed by Dorfi \& Feuchtinger (1995) and in several of our previous papers about dust-driven wind models (cf. Höfner et al. 1995 and references therein).

It is a rather complicated problem to test a code which is designed to describe complex astrophysical objects like dynamical atmospheres of cool giants, without reducing the input physics or the initial/boundary conditions to trivial cases. Therefore, we have performed a detailed comparison of the grey version of our models with a code which was developed by Fleischer et al. $(1992,1995)$ for a similar purpose but based on completely different numerical methods. The results of this comparison can be found in Höfner et al. (1996).
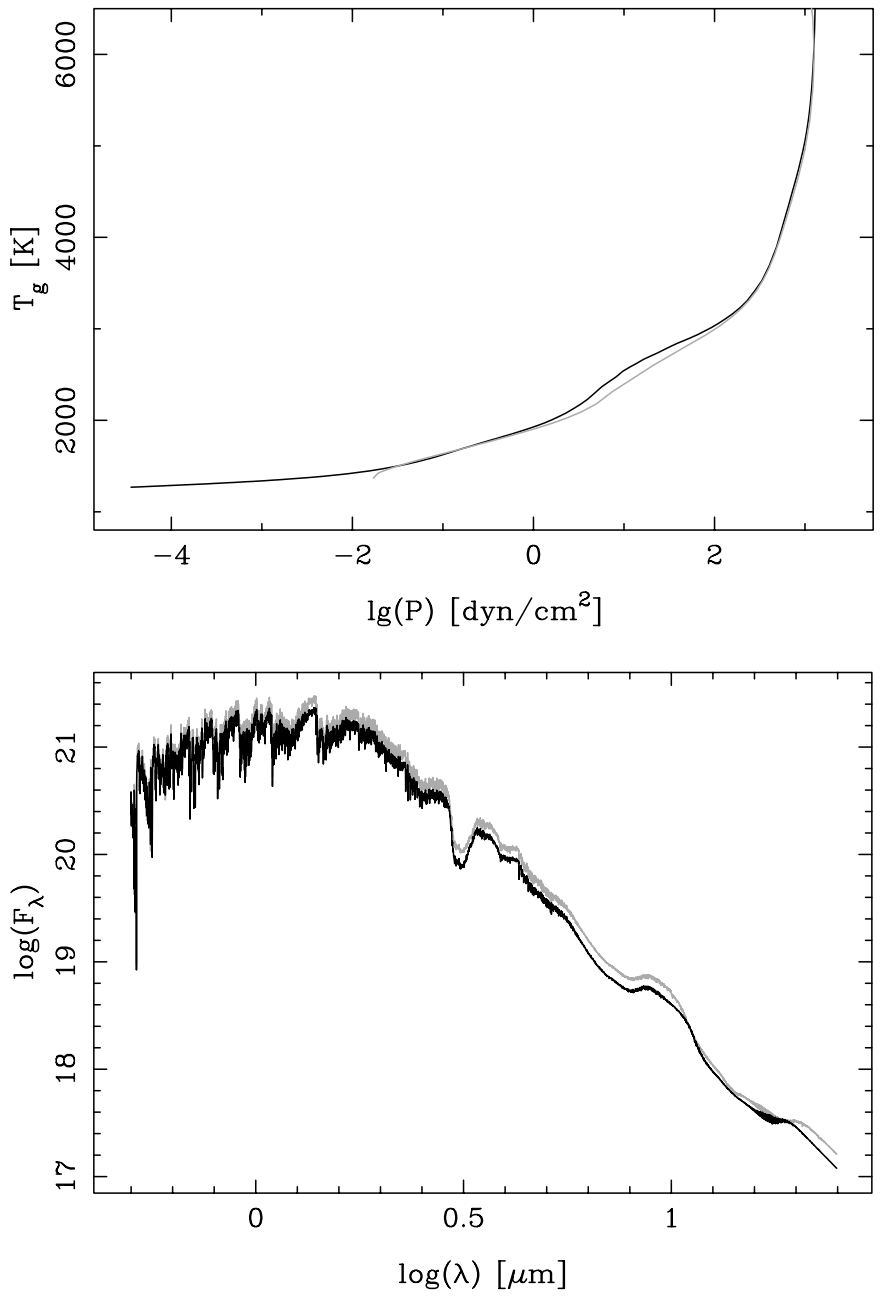

Fig. 1. Temperature-pressure structures (upper panel) and the corresponding spectra (lower panel) of the hydrostatic initial model of 152t30c11u2 (black lines) and of a MARCS model (grey lines) with similar stellar parameters (see Table 2). In spite of the large difference in the number of frequency points used in the computations of the atmospheric structures, the two models are quite similar (cf. Sect. 2.3). Note that the flux in the lower panel is in arbitrary units and that the spectra are shifted relative to each other to make differences and similarities visible.

\subsection{Initial models and boundary conditions}

The dynamical computation starts with a hydrostatic initial model which is characterised by the following parameters: luminosity $L_{\star}$, mass $M_{\star}$, effective temperature $T_{\star}$ and the elemental abundances. This initial model corresponds to the hydrostatic limit case of the radiation-hydrodynamical equations, assuming that no dust is present. It can be directly compared to classic model atmospheres.

Figure 1 shows the pressure-temperature structure (upper panel) and the resulting spectra (lower panel) of the initial model of 152t30c11u2 (see Table 2) and of a corresponding hydrostatic model atmosphere calculated with the MARCS code (Gustafsson et al. 1975; version of Jørgensen et al. 1992 with spherical radiative transfer routines from Nordlund 1984). Both models are based on the same opacity data but use a different number of frequency points (51 and about 5000, respectively) 
for computing the structure of the atmosphere. The differences in the structures and the resulting spectra are small, considering the restricted treatment of radiative transfer in our initial model, in particular compared to the errors introduced by using grey radiative transfer (cf. Höfner 1999 and Höfner et al. 1998). This leads us to expect that our dynamical models even when based on as little as 51 frequency points - allow us to make reasonably realistic predictions of observable properties, in contrast to grey models. We are, however, aware that a realistic structure in the hydrostatic limit is only a necessary, not a sufficient condition for realistic dynamic structures ${ }^{1}$.

The stellar pulsation is simulated by applying a variable inner boundary $R_{\text {in }}(t)$ below the photosphere, moving sinusoidally with a velocity amplitude $\Delta u_{\mathrm{p}}$ and a period $P$. No mass flow is allowed across this inner boundary, forcing the gas close to $R_{\text {in }}$ to move with the boundary. The luminosity at the inner boundary varies like $L_{\text {in }}(t) \propto R_{\text {in }}^{2}(t)$, corresponding to the assumption that the total radiative flux at the inner boundary is constant (see Höfner \& Dorfi 1997). Together, the two boundary conditions $R_{\mathrm{in}}(t)$ and $L_{\mathrm{in}}(t)$ simulate a mass shell in the stellar interior which moves outwards as the luminosity increases and inwards as it decreases during the pulsation. The coupling of the two boundary conditions with the assumption of constant radiative flux in this mass shell reduces the number of free parameters.

In the hydrostatic initial models the outer boundary is located close to the photosphere (typically below $1.5 R_{\star}$ ), due to the steep atmospheric density gradient. When the dynamical calculation is started, the shocks created by the pulsation and the radiation pressure on newly formed dust grains will cause the upper atmosphere to expand. At this stage the outer boundary follows the movement of the gas. If the model develops a wind, the expansion is followed up to a distance of about $20-30 R_{\star}$ where the boundary is fixed, allowing outflow. At this distance the time-averaged wind properties (outflow velocity, degree of condensation, etc.) have usually reached their asymptotic values. In models which do not form a stellar wind the outer boundary moves along with the upper atmospheric layers during the whole computation.

\section{Structure and dynamics}

Regarding their dynamical behaviour, the C-rich models listed in Table 2 fall into two groups: if the mechanical energy input by the pulsation - and therefore the levitation of the outer layers of the atmosphere - is too small no dust will form. In this case, after a transient phase of adjusting from the initial hydrostatic structure, the models settle into a periodic dynamical

\footnotetext{
${ }^{1}$ When selecting the number of frequencies one has to consider the trade-off between the increase in CPU time (which is dominated by the radiative transfer) and the improvements of the resulting models compared to existing grey dynamical models. Our choice of the actual value was guided by the studies of Helling \& Jørgensen (1998) who investigated in detail the errors introduced in hydrostatic MARCS models by picking successively smaller numbers of frequency points and comparing the resulting atmospheric structures with models based on mean opacities. We have started similar studies for our dynamical models and the results will be presented in a future paper.
}

behaviour without forming a stellar wind. On the other hand, if the densities in the cool outer layers are high enough to allow for grain formation an outflow driven by radiation pressure on dust evolves. The O-rich models (Table 3) show a similar division concerning their dynamical properties, depending on whether a (high enough) parameterised dust opacity is included or not.

In the following discussion, we start with dust-free models which do not develop winds to demonstrate the non-grey effects of the gas component. Then we proceed to models which form dusty outflows, discussing the frequency-dependent effects concerning the dust component.

\subsection{Dust-free dynamical atmospheres}

Model 170t28c14u2 is representative of the first group: the levitation of the outer layers is noticeable compared to the hydrostatic case but not sufficient to allow dust grains to form. Figure 2 (top panel) shows the movement of individual mass layers in this model as a function of time after the model has reached its limit cycle behaviour. Each pulsation period the movement of the deep atmospheric layers triggers the formation of a shock wave. The shock (seen as the sharp bends in the trajectories during the last quarter of each period) propagates outwards through the atmosphere, steepening in the process, and losing energy by radiation. After a layer is hit by a shock wave it follows a roughly ballistic trajectory (modified by other forces than gravity, due to gas and radiation pressure) and returns to its initial position before being hit by the next shock wave.

Figure 3 shows the radial structure of model $170 \mathrm{t} 28 \mathrm{c} 14 \mathrm{u} 2$ at different phases (dashed, dash-dotted, dotted and dash-tripledotted lines) and of the corresponding hydrostatic initial model (full line). Panel (a) shows the gas velocity: the newly-formed shock (dashed) moves outwards and becomes stronger (dashdotted), and finally crosses the outer boundary (dotted). After that the material in the outer layers starts to fall back while the next shock is forming below the photosphere (dash-tripledotted). The gas density (panel b) which shows steep steps at the location of shocks is much higher in the outer layers than in corresponding layers of the hydrostatic model (full line). Note that at no instant the density or temperature structures (panel c) resemble the hydrostatic model.

Panel (d) of Fig. 3 shows the ratio of the radiative equilibrium gas temperature to the radiation temperature, i.e.

$$
\left(\frac{T_{\mathrm{g}}}{T_{\mathrm{r}}}\right)^{\mathrm{eq}}=\left(\frac{\kappa_{\mathrm{J}}}{\kappa_{\mathrm{S}}}\right)^{1 / 4} \longleftrightarrow \kappa_{\mathrm{J}} J-\kappa_{\mathrm{S}} S\left(T_{\mathrm{g}}^{\mathrm{eq}}\right)=0
$$

where $S\left(T_{\mathrm{g}}^{\mathrm{eq}}\right)=B\left(T_{\mathrm{g}}^{\mathrm{eq}}\right)$ and $T_{\mathrm{r}}$ is defined by Eq. (18). This plot is a measure for the importance of non-grey radiative transfer. For a grey model, by definition, this ratio would be unity everywhere. The non-grey models, however, show strong deviations from this value in the outer layers due to the influence of molecular opacities. The complex molecules forming in the cooler layers above the photosphere absorb mostly in the nearand mid-infrared, i.e. at wavelengths larger than the flux maximum, leading to a significant cooling of these layers below the 
Table 2. Model parameters $\left(L_{\star}, M_{\star}, T_{\star}, \varepsilon_{\mathrm{C}} / \varepsilon_{\mathrm{O}}, P, \Delta u_{\mathrm{p}}\right)$ and results for C-rich models: mass loss rate $\dot{M}$, mean velocity at the outer boundary $\langle u\rangle$, mean degree of condensation at the outer boundary $\left\langle f_{\mathrm{c}}\right\rangle ; R_{\star}$ is the stellar radius of the hydrostatic initial model (calculated from $L_{\star}$ and $T_{\star}$ ) and, for convenience, we also list the corresponding $\lg g_{\star}$. The dust-to-gas ratio $\rho_{\mathrm{d}} / \rho_{\mathrm{g}}$ is calculated from $f_{\mathrm{c}}$ as described in Höfner \& Dorfi (1997) involving $\varepsilon_{C} / \varepsilon_{O}$ as the only model-dependent quantity.

\begin{tabular}{|c|c|c|c|c|c|c|c|c|c|c|c|c|}
\hline Model & $\begin{array}{l}L_{\star} \\
{\left[L_{\odot}\right]}\end{array}$ & $\begin{array}{l}M_{\star} \\
{\left[M_{\odot}\right]}\end{array}$ & $\begin{array}{l}T_{\star} \\
{[\mathrm{K}]}\end{array}$ & $\begin{array}{l}R_{\star} \\
{\left[R_{\odot}\right]}\end{array}$ & $\lg g_{\star}$ & $\mathrm{C} / \mathrm{O}$ & $\begin{array}{l}P \\
{[\mathrm{~d}]}\end{array}$ & $\begin{array}{l}\Delta u_{\mathrm{p}} \\
{\left[\mathrm{km} \mathrm{s}^{-1}\right]}\end{array}$ & $\begin{array}{l}\dot{M} \\
{\left[\mathrm{M}_{\odot} / \mathrm{yr}\right]}\end{array}$ & $\begin{array}{l}\langle u\rangle \\
{\left[\mathrm{km} \mathrm{s}^{-1}\right]}\end{array}$ & $\left\langle f_{\mathrm{c}}\right\rangle$ & $\begin{array}{l}\rho_{\mathrm{d}} / \rho_{\mathrm{g}} \\
{\left[10^{-3}\right]}\end{array}$ \\
\hline $152 \mathrm{t} 30 \mathrm{c} 11 \mathrm{u} 2$ & 5200 & 1.0 & 3000 & 267 & -0.41 & 1.10 & 295 & 2.0 & - & - & - & - \\
\hline $170 t 30 \mathrm{c} 11 \mathrm{u} 2$ & 7000 & 1.0 & 3000 & 310 & -0.54 & 1.10 & 390 & 2.0 & - & - & - & - \\
\hline $170 \mathrm{t} 28 \mathrm{c} 11 \mathrm{u} 2$ & 7000 & 1.0 & 2800 & 355 & -0.66 & 1.10 & 390 & 2.0 & - & - & - & - \\
\hline $170 t 28 \mathrm{c} 11 \mathrm{u} 4$ & 7000 & 1.0 & 2800 & 355 & -0.66 & 1.10 & 390 & 4.0 & - & - & - & - \\
\hline $170 t 30 \mathrm{c} 14 \mathrm{u} 2$ & 7000 & 1.0 & 3000 & 310 & -0.54 & 1.40 & 390 & 2.0 & - & - & - & - \\
\hline $170 \mathrm{t} 30 \mathrm{c} 14 \mathrm{u} 4$ & 7000 & 1.0 & 3000 & 310 & -0.54 & 1.40 & 390 & 4.0 & - & - & - & - \\
\hline $170 \mathrm{t} 28 \mathrm{c} 14 \mathrm{u} 2$ & 7000 & 1.0 & 2800 & 355 & -0.66 & 1.40 & 390 & 2.0 & - & - & - & - \\
\hline $170 \mathrm{t} 28 \mathrm{c} 14 \mathrm{u} 4$ & 7000 & 1.0 & 2800 & 355 & -0.66 & 1.40 & 390 & 4.0 & $2.4 \times 10^{-6}$ & 11 & 0.28 & 0.63 \\
\hline $170 t 28 c 14 u 5$ & 7000 & 1.0 & 2800 & 355 & -0.66 & 1.40 & 390 & 5.0 & $3.5 \times 10^{-6}$ & 15 & 0.33 & 0.75 \\
\hline $110 \mathrm{t} 28 \mathrm{c} 14 \mathrm{u} 2$ & 10000 & 1.0 & 2800 & 425 & -0.82 & 1.40 & 525 & 2.0 & - & - & - & - \\
\hline $110 \mathrm{t} 28 \mathrm{c} 14 \mathrm{u} 4$ & 10000 & 1.0 & 2800 & 425 & -0.82 & 1.40 & 525 & 4.0 & $2.5 \times 10^{-6}$ & 14 & 0.22 & 0.50 \\
\hline $110 \mathrm{t} 26 \mathrm{c} 14 \mathrm{u} 2$ & 10000 & 1.0 & 2600 & 493 & -0.94 & 1.40 & 525 & 2.0 & $3.6 \times 10^{-6}$ & 18 & 0.30 & 0.68 \\
\hline $110 \mathrm{t} 26 \mathrm{c} 14 \mathrm{u} 4$ & 10000 & 1.0 & 2600 & 493 & -0.94 & 1.40 & 525 & 4.0 & $5.9 \times 10^{-6}$ & 18 & 0.37 & 0.84 \\
\hline $113 \mathrm{t} 27 \mathrm{c} 14 \mathrm{u} 4$ & 13000 & 1.0 & 2700 & 521 & -0.99 & 1.40 & 650 & 4.0 & $8.2 \times 10^{-6}$ & 18 & 0.31 & 0.70 \\
\hline $113 \mathrm{t} 27 \mathrm{c} 14 \mathrm{u} 6$ & 13000 & 1.0 & 2700 & 521 & -0.99 & 1.40 & 650 & 6.0 & $1.1 \times 10^{-5}$ & 17 & 0.31 & 0.70 \\
\hline
\end{tabular}

Grey models based on Planck-mean gas opacities (from Höfner et al. 1998):

$\begin{array}{lllllllllllll}\mathrm{P} 10 \mathrm{C} 14 \mathrm{U} 4 & 10000 & 1.0 & 2790 & 428 & -0.82 & 1.40 & 525 & 4.0 & 6.6 \times 10^{-7} & (3) & 0.19 & 0.43 \\ \mathrm{P} 13 \mathrm{C} 14 \mathrm{U} 4 & 13000 & 1.0 & 2700 & 521 & -0.99 & 1.40 & 650 & 4.0 & 2.9 \times 10^{-6} & 11 & 0.25 & 0.57 \\ \mathrm{P} 13 \mathrm{C} 14 \mathrm{U} 6 & 13000 & 1.0 & 2700 & 521 & -0.99 & 1.40 & 650 & 6.0 & 5.5 \times 10^{-6} & 12 & 0.28 & 0.63\end{array}$

radiation temperature $T_{\mathrm{r}}$ (up to several hundred Kelvin). This intrinsically frequency-dependent effect can not be reproduced by grey models, even if they contain mean molecular opacities.

Since we use the Planck function as source function, the opacity $\kappa_{\mathrm{S}}$ is equal to the Planck mean. Figure $3 \mathrm{~d}$ therefore also gives an impression of how a difference of the actual opacity $\kappa_{\mathrm{J}}$ from the Planck mean affects the atmosphere's energy balance. In the previous papers of this series we used the Planck mean to represent both $\kappa_{\mathrm{J}}$ and $\kappa_{\mathrm{H}}$. However, also the flux-mean opacity $\kappa_{\mathrm{H}}$ differs considerably from the Planck mean in the outer layers of the atmosphere (see Fig. 3 in Höfner 1999). As we already argued in the first paper of this series (Höfner et al. 1998), the Planck mean tends to over-estimate the actual flux mean $\kappa_{\mathrm{H}}$, leading to a too high radiation pressure on the gas. Therefore, the grey models based on Planck means show a lower gas pressure and density at a given temperature than the corresponding frequency-dependent models. This holds throughout the whole atmosphere but the effect is most pronounced in the outer atmospheric layers.
In summary, we find that the frequency-dependent models have cooler and denser upper layers which affects the efficiency of dust condensation and consequently the formation of stellar winds. Next, we will look at how the dust component itself is influenced by non-grey effects.

\subsection{Dust-driven stellar winds}

Model 170t28c14u4 starts from the same initial model as $170 \mathrm{t} 28 \mathrm{c} 14 \mathrm{u} 2$ but uses a larger piston velocity amplitude. In this case, the outer atmospheric layers are cool and dense enough to provide an environment for efficient dust formation. The radiation pressure on the grains drives a stellar wind with a velocity of $11 \mathrm{~km} \mathrm{~s}^{-1}$ and an average mass loss rate of about $2 \times 10^{-6} M_{\odot} / \mathrm{yr}$.

The dust formation cycle in this model spans two pulsation periods: every other period a dust shell is created and the spatial pattern of the model repeats on this time scale. This behaviour can nicely be seen in Fig. 2 (lower panel): the shocks 
Table 3. Model parameters $\left(L_{\star}, M_{\star}, T_{\star}, \varepsilon_{\mathrm{C}} / \varepsilon_{\mathrm{O}}, P, \Delta u_{\mathrm{p}} ; \kappa_{\mathrm{d}}^{\max }\right)$ and results for O-rich models: mass loss rate $\dot{M}$, mean velocity at the outer boundary $\langle u\rangle ; R_{\star}$ is the stellar radius of the hydrostatic initial model (calculated from $L_{\star}$ and $T_{\star}$ ) and, for convenience, we also list the corresponding $\lg g_{\star}$.

\begin{tabular}{|c|c|c|c|c|c|c|c|c|c|c|c|}
\hline Model & $\begin{array}{l}L_{\star} \\
{\left[L_{\odot}\right]}\end{array}$ & $\begin{array}{l}M_{\star} \\
{\left[M_{\odot}\right]}\end{array}$ & $\begin{array}{l}T_{\star} \\
{[\mathrm{K}]}\end{array}$ & $\begin{array}{l}R_{\star} \\
{\left[R_{\odot}\right]}\end{array}$ & $\lg g_{\star}$ & $\mathrm{C} / \mathrm{O}$ & $\begin{array}{l}P \\
{[\mathrm{~d}]}\end{array}$ & $\begin{array}{l}\Delta u_{\mathrm{p}} \\
{\left[\mathrm{km} \mathrm{s}^{-1}\right]}\end{array}$ & $\begin{array}{l}\kappa_{\mathrm{d}}^{\max } \\
{\left[\mathrm{cm}^{2} / \mathrm{g}\right]}\end{array}$ & $\begin{array}{c}\dot{M} \\
{\left[\mathrm{M}_{\odot} / \mathrm{yr}\right]}\end{array}$ & $\begin{array}{c}\langle u\rangle \\
{\left[\mathrm{km} \mathrm{s}^{-1}\right]}\end{array}$ \\
\hline 150t28osu3 & 5000 & 1.0 & 2800 & 300 & -0.51 & 0.48 & 310 & 3.0 & - & - & - \\
\hline $150 \mathrm{t} 28 \mathrm{osu} 3 \mathrm{kd} 3$ & 5000 & 1.0 & 2800 & 300 & -0.51 & 0.48 & 310 & 3.0 & 3 & $3.7 \times 10^{-7}$ & 13 \\
\hline $110 \mathrm{t} 28 \mathrm{osu} 2$ & 10000 & 1.0 & 2800 & 425 & -0.82 & 0.48 & 525 & 2.0 & - & - & - \\
\hline $110 \mathrm{t} 28 \mathrm{osu} 2 \mathrm{kd} 2$ & 10000 & 1.0 & 2800 & 425 & -0.82 & 0.48 & 525 & 2.0 & 2 & $2.7 \times 10^{-6}$ & 17 \\
\hline $110 \mathrm{t} 28 \mathrm{osu} 2 \mathrm{kd} 3$ & 10000 & 1.0 & 2800 & 425 & -0.82 & 0.48 & 525 & 2.0 & 3 & $3.2 \times 10^{-6}$ & 24 \\
\hline $110 \mathrm{t} 28 \mathrm{osu} 2 \mathrm{kd} 5$ & 10000 & 1.0 & 2800 & 425 & -0.82 & 0.48 & 525 & 2.0 & 5 & $3.7 \times 10^{-6}$ & 33 \\
\hline
\end{tabular}

created around period 100, 102 and 104 trigger dust formation and the radiation pressure drives the material in the wake of these shocks away from the star. The shock waves originating at period 101 and 103 on the other hand are not followed by the formation of a new dust shell. Meeting in-falling material from the previous period, these shocks slow down, the gas is starting to fall inwards and is finally overrun by the next propagating shock wave.

Figure 4 shows the radial structure of model $170 \mathrm{t} 28 \mathrm{c} 14 \mathrm{u} 4$ at different phases (dashed, dash-dotted, dotted and dash-tripledotted lines) and of the corresponding hydrostatic initial model (full line). The same quantities are plotted here as in Fig. 3 for the model with the smaller pulsation amplitude (note however the different radius ranges). The velocity structure (panel a) shows strong shocks with both positive and negative radial velocities in the innermost part of the model ${ }^{2}$. Around $2-3 R_{\star}$ (dust formation zone) the profiles change to a continuous outflow with superimposed small-amplitude shocks. The density structure (panel b) shows steep steps at the location of strong shocks in the inner zones of the model and a transition to a $1 / r^{2}$ profile in the outer layers (note that the actual computational domain stretches about 3 times further out than in these plots).

\subsubsection{Temperatures of gas and dust}

Panel (d) of Fig. 4 shows the ratio of the radiative equilibrium gas temperature to the radiation temperature. As in model $170 \mathrm{t} 28 \mathrm{c} 14 \mathrm{u} 2$, the equilibrium gas temperature is considerably lower than the radiation temperature due to the cooling by molecules. The overall effect is somewhat smaller than in the dust-free model (probably due to the effects of the continuous dust opacity; backwarming). The largest non-grey effects are, however, seen in the critical zone around $2 R_{\star}$ where dust formation starts (see degree of condensation, top panel in Fig. 5) and where a significant fraction of the infrared molecular features is formed. Therefore the non-grey treatment of radiative

\footnotetext{
${ }^{2}$ Note that positive velocities mean outflow and negative ones inflow relative to the stellar center, in contrast to observations which often use the opposite notation.
}

transfer has a considerable influence both on the resulting spectra and the stellar wind.

Panel (c) of Fig. 5 shows the corresponding plot for the dust temperature. The deviation from the radiation temperature is smaller than for the gas but goes into the opposite direction: the dust grains are hotter than the radiation temperature and considerably hotter than the gas. This is due to the fact that the opacity of amorphous carbon grains is roughly proportional to $1 / \lambda$. Therefore the dust grains absorb and emit radiation preferentially at short wavelengths. This property in combination with the radiative equilibrium condition requires the grains to be hotter than the radiation temperature in order to emit as much energy as they absorb ${ }^{3}$.

To demonstrate this effect more clearly we have assumed different functions describing the wavelength dependence of the absorption efficiency as shown in Fig. 6 (top). The full line represents the data of Rouleau \& Martin (1991) used in the dynamical calculations. The dotted line is a simple $1 / \lambda$ power law which closely resembles the actual data in the critical region around $1 \mu \mathrm{m}$ and the dash-dotted line is a $1 / \lambda^{2}$ power law. The dashed line represents the grey case. The lower part of this figure shows the corresponding equilibrium grain temperatures for a phase of model $170 \mathrm{t} 28 \mathrm{c} 14 \mathrm{u} 4$. The full line is the actual value in the dynamical calculation. The dotted line follows this curve closely, reflecting the similar wavelength dependence of the absorption efficiency. The steeper power law (dash-dotted) leads to a much higher temperature. The grey value (dashed) on the other hand produces grain temperatures equal to the radiation temperature, i.e. smaller values than the actual temperatures in the model.

\subsubsection{Radiative pressure and mass loss}

In addition to its effects on the temperatures of gas and dust grains the frequency-dependent description of radiative transfer also influences the momentum balance of the atmosphere and wind. In Sect. 3.1 we have described the effect of the

\footnotetext{
3 Note that the radiation temperature $T_{\mathrm{r}}$ is not a temperature in the sense of gas kinetics but a measure of the energy contained in the local radiation field; see definition, Eq. (18).
} 

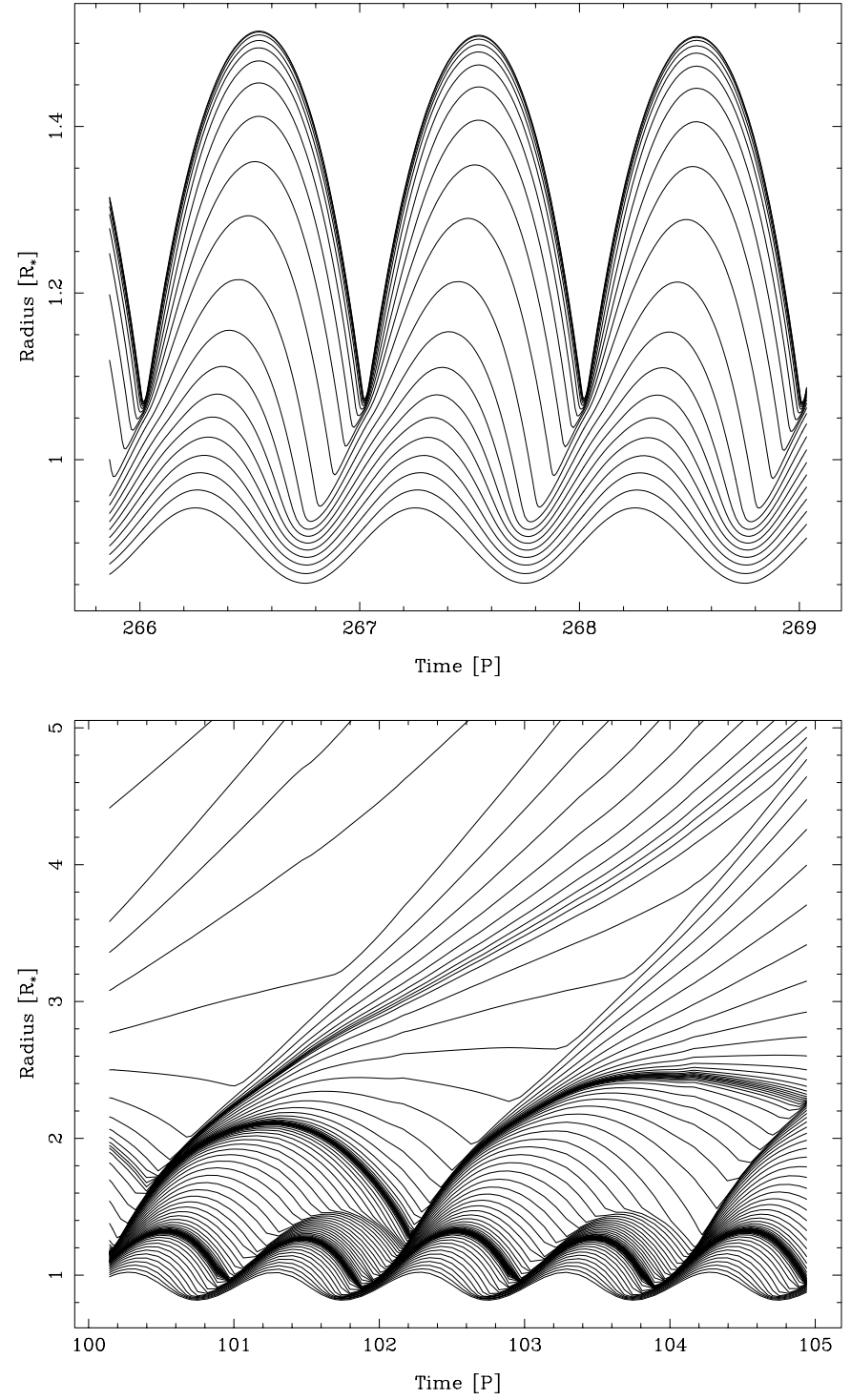

Fig. 2. Position of selected mass shells as a function of time for models $170 \mathrm{t} 28 \mathrm{c} 14 \mathrm{u} 2$ (top) and 170t28c14u4 (bottom); time in piston periods $P$, radius in units of the radius of the hydrostatic initial model (note the different radius ranges). The two models have identical parameters except for the piston velocity amplitude $\Delta u_{\mathrm{p}}$. In model 170t28c14u2 $\left(\Delta u_{\mathrm{p}}=2 \mathrm{~km} \mathrm{~s}^{-1}\right.$; upper panel) the levitation of the atmosphere is not strong enough to cause dust formation and mass loss, while model $170 \mathrm{t} 28 \mathrm{c} 14 \mathrm{u} 4\left(\Delta u_{\mathrm{p}}=4 \mathrm{~km} \mathrm{~s}^{-1}\right.$; lower panel) develops a dust-driven outflow. Note that the selection of the trajectories does not represent, e.g., the gas density or other physical properties of the model but is due to the fact that the locations of actual grid points at some instant where used as starting points to trace the movement of matter. The distribution of these grid points reflects the property of the adaptive grid to resolve steep gradients. In this sense the selection of tracks is 'random' for the purpose of this figure. Notice further, that the time resolution in the plots is limited by the (sometimes quite large) time steps in the model computation.

radiation pressure on the gas density. Figure $5 \mathrm{~d}$ shows the ratio of the actual flux-mean dust opacity (which determines the radiative pressure on the dust grains in the dynamical calculation) to the value resulting from the gray opacity used in previous models. The figure demonstrates that the actual radiative
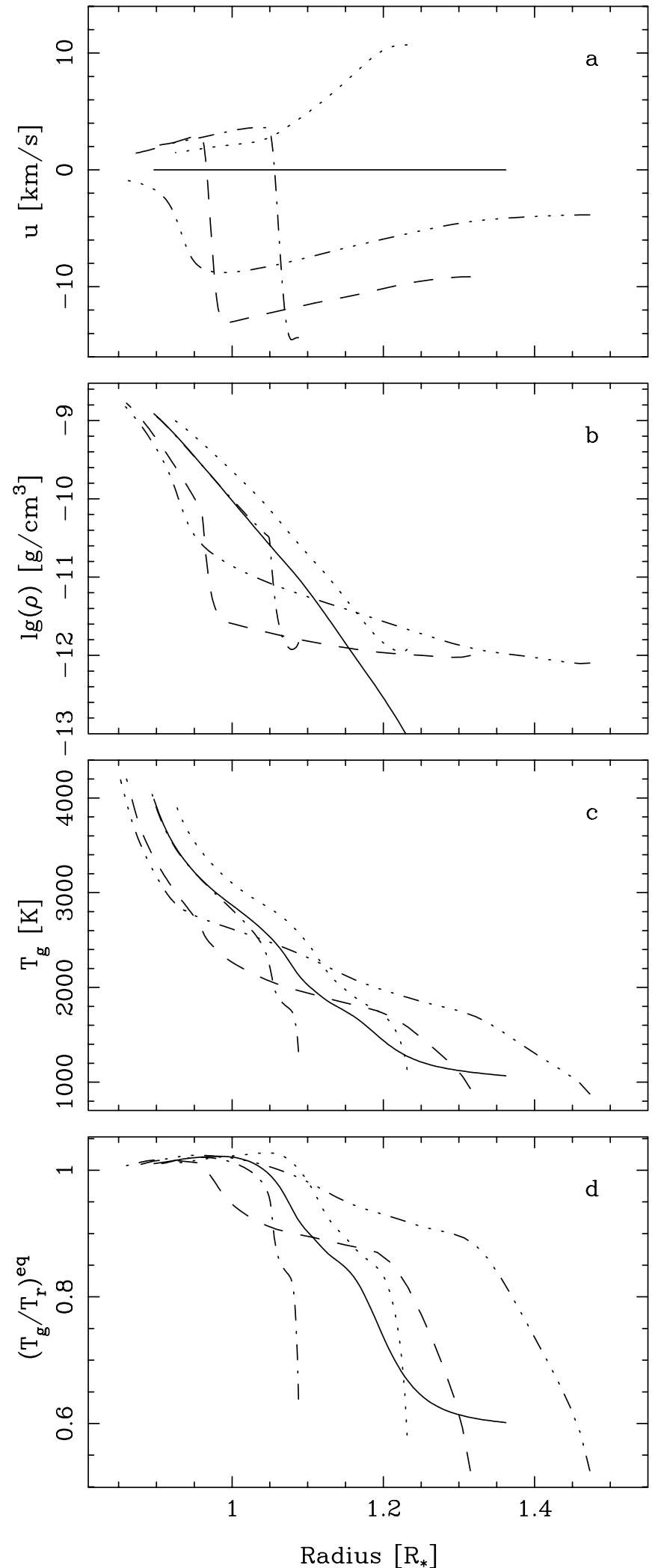

Fig. 3. Radial structure of model 170t28c14u2 (see also upper panel of Fig. 2) at different phases (dashed, dash-dotted, dotted and dashtriple-dotted) and of the corresponding hydrostatic initial model (full line); flow velocity a), gas density b), gas temperature c) and the ratio of the radiative equilibrium gas temperature to the radiation temperature d) as a function of the radius (given in units of the radius of the hydrostatic initial model). 

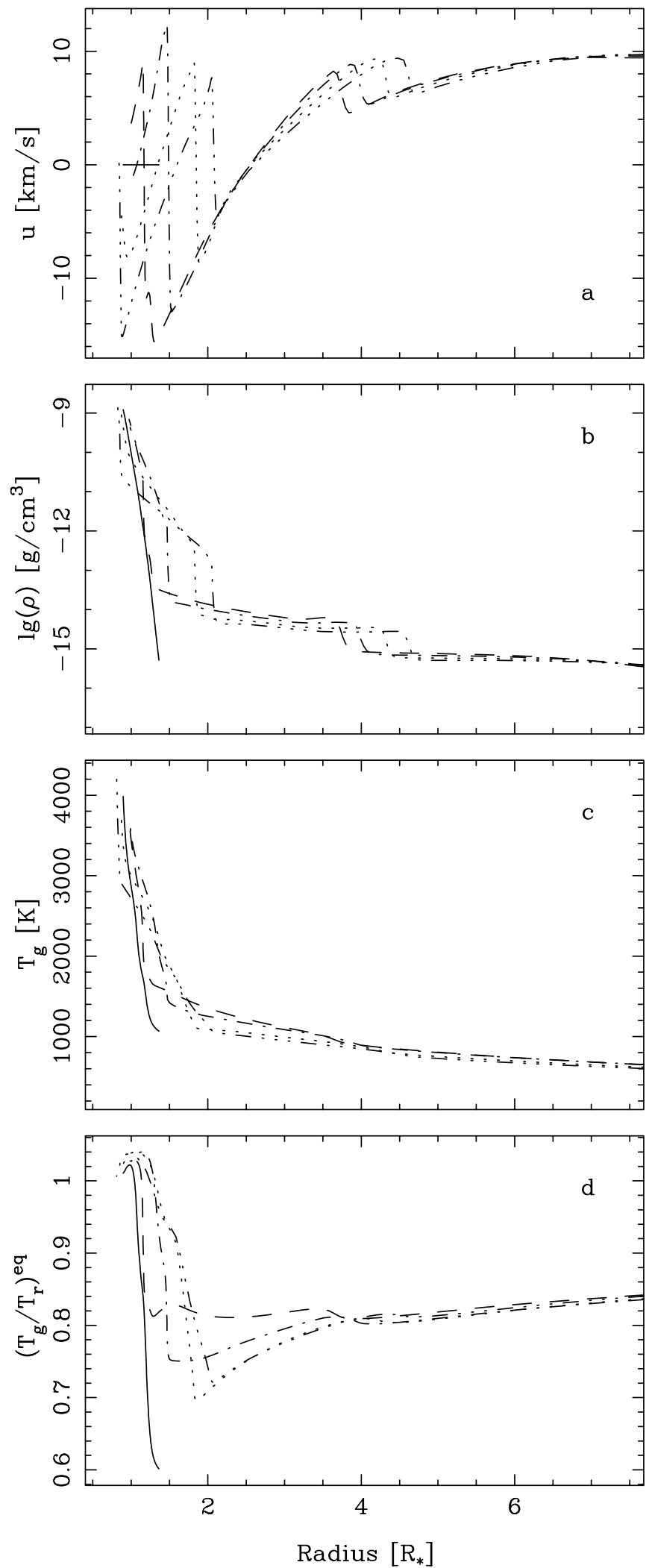

Fig. 4. Radial structure of model 170t28c14u4 (see also lower panel of Fig. 2) at different phases (dashed, dash-dotted, dotted and dashtriple-dotted) and of the corresponding hydrostatic initial model (full line); flow velocity a), gas density b), gas temperature c) and the ratio of the radiative equilibrium gas temperature to the radiation temperature d) as a function of the radius (given in units of the radius of the hydrostatic initial model).
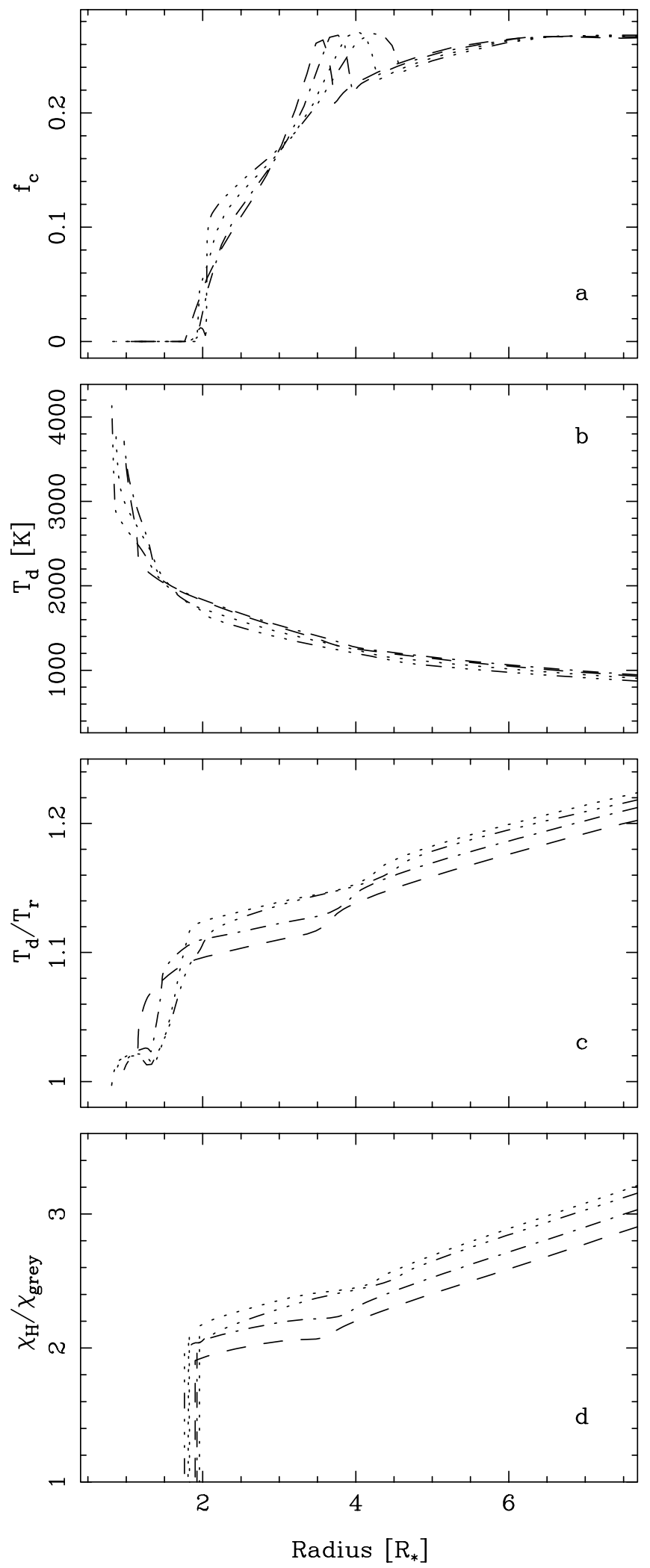

Fig. 5. Radial structure of model 170t28c14u4 (see also lower panel of Fig. 2) at different phases (dashed, dash-dotted, dotted and dash-tripledotted); degree of condensation a), dust temperature b), the ratio of the dust temperature to the radiation temperature c) and the ratio of the flux-mean dust opacity to the grey value used in previous models d) as a function of the radius (given in units of the radius of the hydrostatic initial model). 

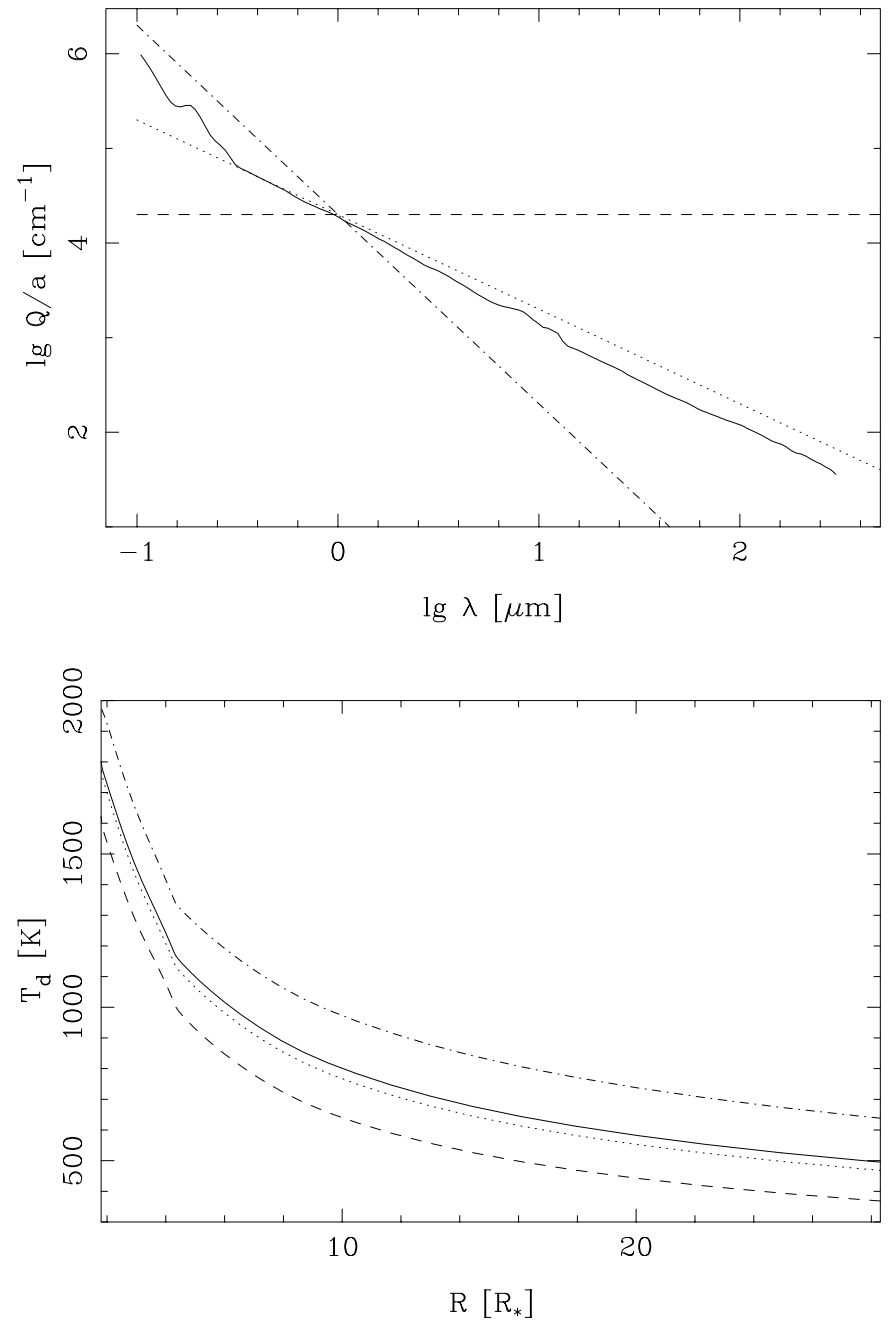

Fig. 6. Upper panel: wavelength dependence of the dust absorption efficiency: data from Rouleau \& Martin (1991; full line), power laws with $Q_{\text {ext }}^{\prime} \propto 1 / \lambda$ (dotted), $Q_{\text {ext }}^{\prime} \propto 1 / \lambda^{2}$ (dash-dotted) and a constant value (grey opacity; dashed line); lower panel: resulting equilibrium grain temperatures for a phase of model $170 \mathrm{t} 28 \mathrm{c} 14 \mathrm{u} 4$. The full line is the actual value in the dynamical calculation and the other lines correspond to the opacities in the upper panel with the same line style.

pressure using the flux mean of the grain opacity is $2-3$ times larger in the wind acceleration zone than the grey value (assuming otherwise identical properties of the dust and the radiation field).

The conclusion that the radiative pressure is accelerating the outflow more efficiently in the new frequency-dependent C-star models is also supported by a comparison with the grey wind models presented by Höfner et al. (1998). In Table 2 we have listed three of these models which have combinations of stellar parameters similar to some of our new models. For all three models the mass loss rate and the wind velocity is lower than in the corresponding non-grey models. The differences are largest for models P10C14U4 / 110t28c14u4: the grey model has a very low outflow velocity and a small mass loss rate, indicating that it is close to the threshold in the stellar parameters where a dust-driven wind can form at all. Close to this threshold the mass loss rate increases steeply with decreasing effective temperature, increasing luminosity and increasing carbon abundance (cf. Höfner \& Dorfi 1997). The location of this transition region in terms of stellar parameters is influenced by the effects of frequency-dependent transfer.

Models P13C14U4 / 113t27c14u4 and P13C14U6 / $113 \mathrm{t} 27 \mathrm{c} 14 \mathrm{u} 6$ have a pronounced mass loss with reasonable velocities, both for the grey and the non-grey case. Therefore they give a more balanced picture of the non-grey effects in the radiative pressure. The dust opacity depends both on the optical properties of the grain material as discussed above and on the amount of dust present in the model. The later is represented by the degree of condensation and the dust-to-gas mass ratio given in Table 2 . The non-grey models produce somewhat more dust but the increase in the outflow velocity is larger than the increase in the degree of condensation. Therefore it must be partly caused by a non-grey effect in the flux mean of the dust opacity as shown in Fig. 5d. The differences in the mass loss rates on the other hand probably reflect mostly the overall differences of the gas density in the Planck-mean models compared to the frequency-dependent case as discussed in Sect. 3.1 (cf. Höfner et al. 1998 and Höfner 1999).

\section{Observable properties}

A comprehensive discussion of the observable properties of our new models is beyond the scope of this paper. However, we want to highlight some basic results which demonstrate the improvements achieved compared to grey dynamical models and even to classical hydrostatic model atmospheres.

For a comparison of our carbon-rich dynamical models with observations of AGB stars, we calculated synthetic spectra for models P13C14U4 (based on Planck-mean gas opacities) and $113 \mathrm{t} 27 \mathrm{c} 14 \mathrm{u} 4$ (frequency-dependent radiative transfer). Both models have the same stellar parameters and also the resulting mass loss rates are comparable (see Table 2).

Figure 7 shows (from top to bottom) minimum light spectra of the grey model P13C14U4, the frequency dependent model 113t27c14u4, the carbon-rich SRa variable R Scl (Hron et al. 1998), and the carbon-rich Mira R Lep (Lançon \& Wood 2000). It is evident that the grey model is not able to reproduce the energy distribution of R Lep (bottom curve) correctly; the maximum of its energy distribution is far too blue. The frequency dependent model on the other hand reproduces the overall shape quite well.

Figure 8 shows two different spectral regions. In the left panel we plotted the wavelength range between 0.7 and $1.0 \mu \mathrm{m}$, where $\mathrm{C}_{2}$ and $\mathrm{CN}$ features dominate the spectrum. The main contributions to these features originate from layers where $T>$ $2500 \mathrm{~K}$ (Loidl et al. 2001). The frequency dependent model has a systematically higher gas pressure at a given temperature than the grey model for temperatures larger than $3000 \mathrm{~K}$. This causes the overall shape of the energy distribution to look completely different, but the feature intensities of the $\mathrm{C}_{2}$ and $\mathrm{CN}$ features to be very similar (top and middle curve in the left panel of Fig. 8). The bottom curve on the left side of Fig. 8 shows a spectrum of R Lep for comparison. Our spectra reproduce all features visible in R Lep in this wavelength region; we did not attempt to fit R Lep, however, by fine-tuning the 


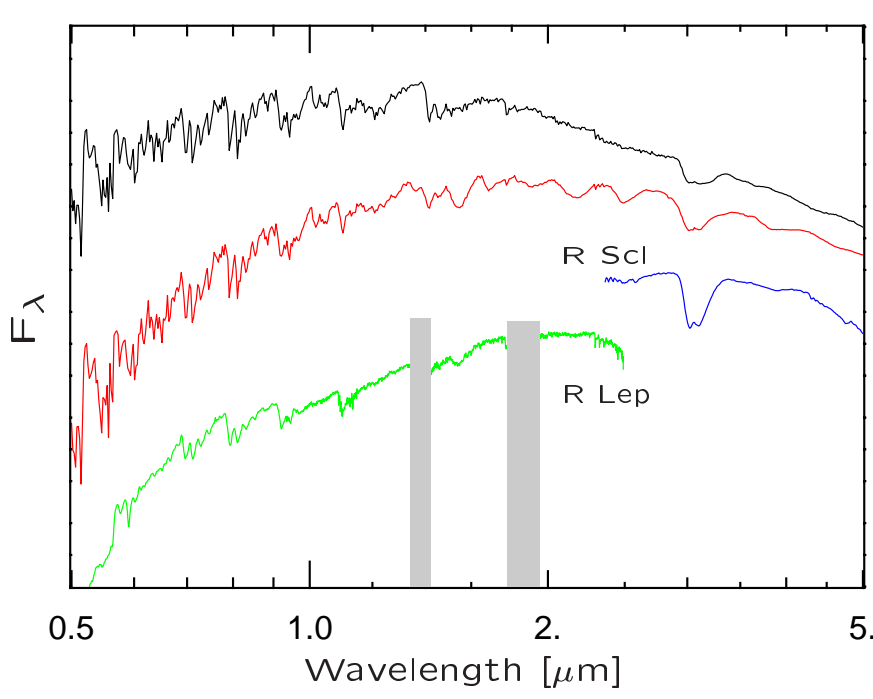

Fig. 7. Synthetic minimum light spectra of the grey model P13C14U4 (top), the frequency dependent model $113 \mathrm{t} 27 \mathrm{c} 14 \mathrm{u} 4$ (upper middle), as well as spectra of R Scl (lower middle) and R Lep (bottom). The grey shaded areas mark regions of the spectrum of $\mathrm{R}$ Lep which are contaminated by the Earth's atmosphere. Note that the frequency-dependent model (second from top) reproduces the overall energy distribution of the observed spectra quite well, in contrast to the grey model with identical parameters (top) which shows a maximum at too short wavelengths. Note further that no detailed fit to the observed spectra was attempted here.

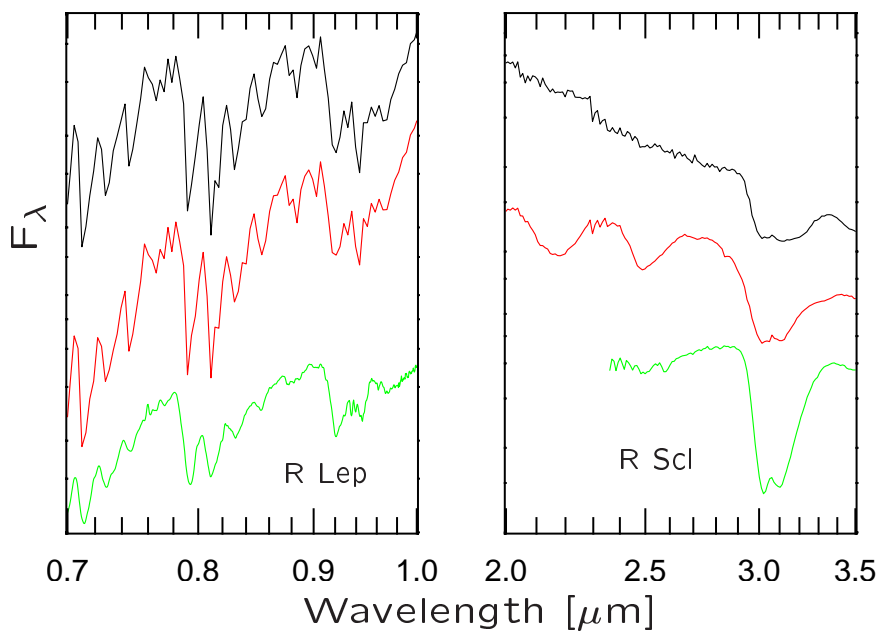

Fig. 8. Left panel: synthetic minimum light spectra of the grey model P13C14U4 (top), the frequency dependent model 113t27c14u4 (middle), and a spectrum of R Lep (bottom) for comparison. Right panel: synthetic minimum light spectra of the grey model P13C14U4 (top), the frequency dependent model 113t27c14u4 (middle), and a spectrum of $\mathrm{R} \mathrm{Scl}$ (bottom) for comparison.

stellar parameters of the model, thus it is not surprizing that the feature intensities are not matched.

In the right panel of Fig. 8 the wavelength range between 2.0 and $3.5 \mu \mathrm{m}$ is plotted for the grey model (top), the frequency dependent model (middle), and for a spectrum of R Scl (bottom). $\mathrm{C}_{2} \mathrm{H}_{2}$ and $\mathrm{HCN}$ features dominate this spectral region; features which are formed mainly at temperatures below $2000 \mathrm{~K}$. The structures of the grey and non-grey models differ considerably below $2000 \mathrm{~K}$ and the high temperature sensitivity of the molecules $\mathrm{C}_{2} \mathrm{H}_{2}$ and $\mathrm{HCN}$ (Jørgensen et al. 2000) results in spectra of model P13C14U4 (grey) and 113t27c14u4 (frequency dependent) which look quite different in this region. The bottom curve on the right side of Fig. 8 shows a spectrum of $\mathrm{R} \mathrm{Scl}$ for comparison, where one can see a weak $2.5 \mu \mathrm{m}$ and a strong $3 \mu \mathrm{m} \mathrm{C}_{2} \mathrm{H}_{2} / \mathrm{HCN}$ band.

The new frequency dependent models are able to describe observed spectra in the wavelength range between 0.5 and $5 \mu \mathrm{m}$, as well as observed near infrared colours of carbon stars (Andersen et al. 2002). At wavelengths greater than $5 \mu \mathrm{m}$ those frequency dependent models which possess a considerable mass loss show emissions of the $\mathrm{C}_{3}, \mathrm{C}_{2} \mathrm{H}_{2}$ and $\mathrm{HCN}$ features where the observed spectra show partly prominent (e.g. $\mathrm{C}_{3}$ feature at $5.1 \mu \mathrm{m}$ ) or weak (e.g. $\mathrm{C}_{2} \mathrm{H}_{2}$ feature around $14 \mu \mathrm{m}$ ) absorption features. The spectra of grey models, however, predict much stronger emissions longwards of $4 \mu \mathrm{m}$ than those of frequency dependent models. A more detailed comparison of dynamical models with observations of carbon-rich AGB stars will be presented in a forthcoming paper.

For oxygen-rich AGB variables the importance of using structures derived from non-grey model calculations is illustrated in Fig. 9. We compare synthetic ISO-SWS spectra derived from MARCS atmospheres to results obtained from our hydrostatic initial models and selected phases of dynamical models with the same or quite similar stellar parameters. The left panel includes the spectrum of the initial model of P5tOU2 (see Höfner et al. 1998 and Aringer et al. 1999) which is based on Planck-mean gas opacities. It has an effective temperature of $2880 \mathrm{~K}$, a luminosity of $5000 L_{\odot}$ and one solar mass. As one can clearly see, the emerging $\mathrm{H}_{2} \mathrm{O}$ absorption in the $2.5 \mu \mathrm{m}$ region is significantly weaker than in the corresponding MARCS model. In fact, in the dynamical atmosphere the water features are almost not visible. The large effects appearing mainly for the water bands are due to the fact that this molecule dominates the overall opacity in the infrared range of cool M-type objects, making the intensities of the features more sensitive to an inconsistent (in this case grey) determination of the atmospheric structure. Compared to the existing ISO-SWS observations of oxygen-rich AGB variables, the results based on grey atmospheres are completely unrealistic: as it was shown by Aringer et al. (2002b) or Tsuji et al. (1999), the observed $2.5 \mu \mathrm{m}$ water depression is in many cases even much stronger than predicted by the hydrostatic MARCS models. Aringer et al. claim that this is most probably due to dynamical effects. However, the discussed grey dynamical model gives much weaker features.

The right panel shows synthetic spectra based on a MARCS model and two phases of the dynamical atmosphere 110t28osu2 (see Table 3, model without dust kappa) which was computed using non-grey radiative transfer. For the dynamical model, the water features are more intense than for the hydrostatic MARCS model, in significantly better agreement with the observations. It should be noted that the used model still cannot reproduce the deep water depression observed by Aringer et al. (2002b) or Matsuura et al. (2002) in Mira variables around minimum light. This, however, may be due to the parameters of the chosen model. Currently, we are performing a more detailed study of our O-rich models regarding the water features, and 


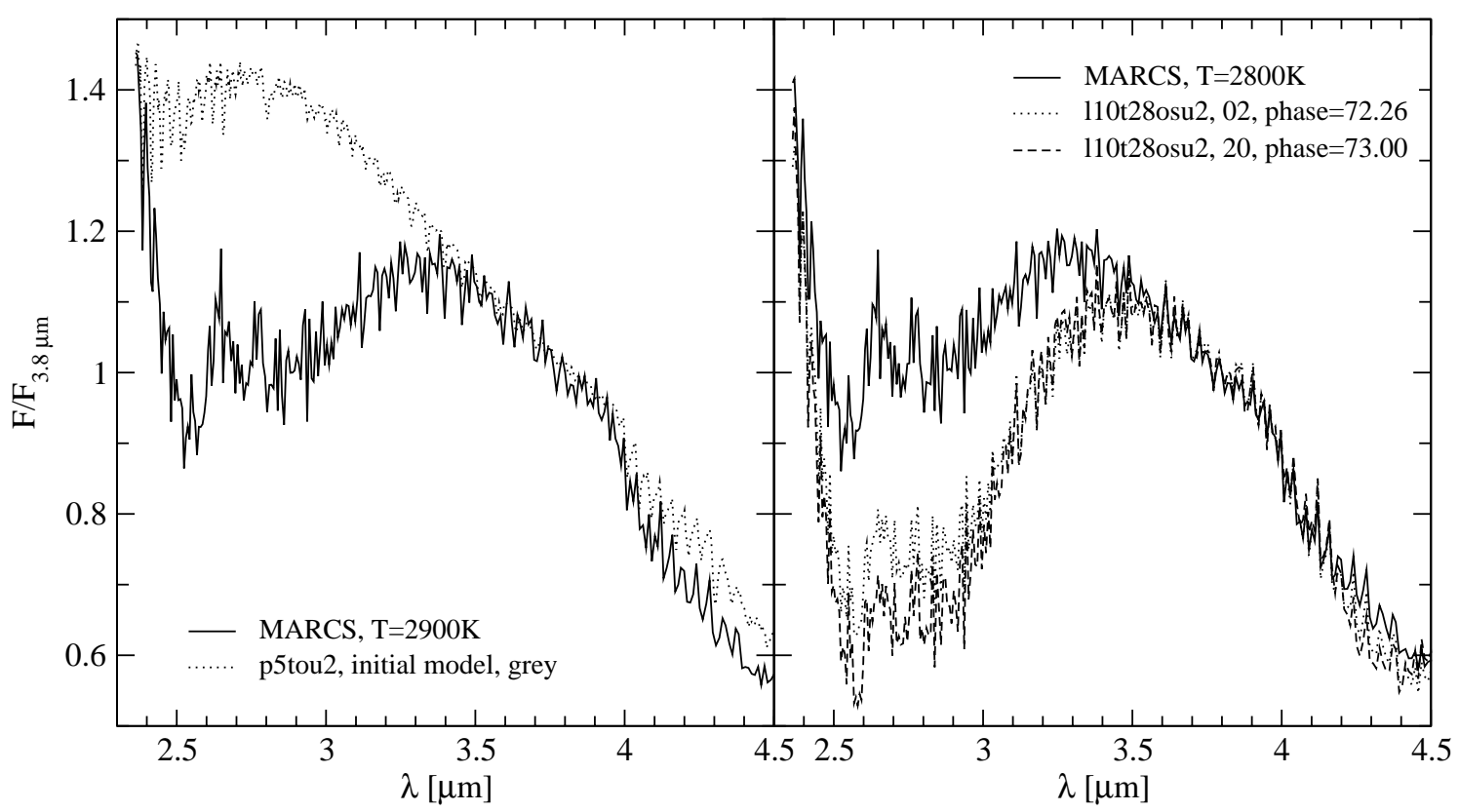

Fig. 9. Left panel: a synthetic ISO-SWS spectrum based on the hydrostatic initial model of the grey dynamical atmosphere p5tou2 (dotted) is compared to results obtained from a MARCS model (full line) with similar stellar parameters. Right panel: spectra calculated from two phases of the non-grey atmosphere 110t28osu2 (dotted, dashed) and from the corresponding MARCS model (full line; same parameters as the initial model of 110t28osu2). The water features of the frequency-dependent dynamical model (dotted, dashed) are much more intense than those of the MARCS model (full line) and they are in significantly better agreement with observations. All spectra are normalized to the flux at $3.8 \mu \mathrm{m}$ where the molecular opacity has a minimum and the resolution is set to 450 .

also of the variation of $\mathrm{CO}$ line profiles in both M- and C-type Miras.

\section{Conclusions}

In order to understand atmospheres of pulsating AGB stars it is essential to include time-dependent dynamics in the models. Dynamical processes influence the atmospheric structure both on local and global scales (propagating shock waves, levitation, winds). Sequences of hydrostatic models with varying stellar parameters cannot reproduce these effects. Therefore, dynamical models are the only consistent way of studying temporal variations of observable properties like colors, low-resolution molecular spectra and line profiles, as well as mass loss by stellar winds.

In this paper we have demonstrated that a frequencydependent treatment of radiative transfer in such dynamical models is crucial for obtaining realistic structures and synthetic spectra, and reliable mass loss rates. Grey opacities - even if they include molecular data - lead to systematically wrong global density-temperature structures which affects the resulting spectra and consequently the interpretation of observed data.

Comparing the hydrostatic limit case of our models to welltested MARCS model atmospheres and presenting synthetic spectra, we have demonstrated that the new generation of dynamical models - despite the still rather restricted treatment of radiative transfer - represents an important step in a transition from qualitative to quantitative modelling. More comprehensive studies of observational properties and a detailed comparison to observations are currently performed and will be presented in future papers.
Acknowledgements. This work has been supported by the Swedish Research Council and the Royal Swedish Academy of Sciences. BA is supported by the Austrian Science Fund FWF (grants J2030 and J2216).

\section{References}

Alvarez, R., Lançon, A., Plez, B., \& Wood, P. R. 2000, A\&A, 353, 322

Alvarez, R., \& Plez, B. 1998, A\&A, 330, 1109

Andersen, A. C., Höfner, S., \& Loidl, R. 2002, in Radial and Nonradial Pulsations as Probes of Stellar Physics, ed. C. Aerts, T. Bedding, \& J. Christensen-Dalsgaard, ASP Conf. Ser., 259, 542

Aringer, B., Höfner, S., Wiedemann, G., et al. 1999, A\&A, 342, 799

Aringer, B., Jørgensen, U. G., Kerschbaum, F., Hron, J., \& Höfner, S. 2002a, in Radial and Nonradial Pulsations as Probes of Stellar Physics, ed. C. Aerts, T. Bedding, \& J. Christensen-Dalsgaard, ASP Conf. Ser., 259, 538

Aringer, B., Kerschbaum, F., \& Jørgensen, U. G. 2002b, A\&A, 395, 915

Bowen, G. H. 1988, ApJ, 329, 299

Dorfi, E. A., \& Drury, L. O'C. 1987, J. Comput. Phys., 69, 175

Dorfi, E. A., \& Feuchtinger, M. U. 1995, Comput. Phys. Commun., 89,69

Fleischer, A. J., Gauger, A., \& Sedlmayr, E. 1992, A\&A, 266, 321

Fleischer, A. J., Gauger, A., \& Sedlmayr, E. 1995, A\&A, 297, 543

Gail, H.-P., \& Sedlmayr, E. 1988, A\&A, 206, 153

Gauger, A., Gail, H.-P., \& Sedlmayr, E. 1990, A\&A, 235, 345

Gustafsson, B., Bell, R. A., Eriksson, K., \& Nordlund, А. 1975, A\&A, 42,407

Helling, C., \& Jørgensen, U. G. 1998, A\&A, 337, 477

Höfner, S. 1999, A\&A, 346, L9

Höfner, S., \& Dorfi, E .A. 1997, A\&A, 319, 648

Höfner, S., Feuchtinger, \& M., Dorfi, E. A. 1995, A\&A, 297, 815 
Höfner, S., Fleischer, A. J., Gauger, A., et al. 1996, A\&A, 314, 204

Höfner, S., Jørgensen, U. G., Loidl, R., \& Aringer, B. 1998, A\&A, 340, 497

Höfner, S., Loidl, R., Aringer, B., \& Jørgensen, U. G. 2002, in Radial and Nonradial Pulsations as Probes of Stellar Physics, ed. C. Aerts, T. Bedding, \& J. Christensen-Dalsgaard, ASP Conf. Ser., 259,534

Hron, J., Loidl, R., Höfner, S., et al. 1998, A\&A, 335, L69

Jørgensen, U. G. 1997, in Molecules in Astrophysics: Probes and Processes. Proc. IAU Symp., ed. E. F. van Dishoeck (Kluwer), 178,441

Jørgensen, U. G., Hron, J., \& Loidl, R. 2000, A\&A, 356, 253

Jørgensen, U. G., Johnson, H. R., \& Nordlund 1992, A\&A, 261, 263

Lançon, A., \& Wood, P. 2000, A\&AS, 146, 217

Loidl, R., Höfner, S., Jørgensen, U. G., \& Aringer, B. 1999, A\&A, 342,531
Loidl, R., Hron, J., Jørgensen, U. G., \& Höfner, S. 2000, in ISO beyond the Peaks: The 2nd ISO workshop on analytical spectroscopy, ESA SP-456, pp. 315-318

Loidl, R., Lançon, A., \& Jørgensen, U. G. 2001, A\&A, 371, 1065

Matsuura, M., Yamamura, I., Cami, J., Onaka, T., \& Murakami, H. 2002, A\&A, 383, 972

Nordlund, Å. 1984, in Methods in Radiative Transfer, ed. W. Kalkofen (Cambridge University Press, Cambridge), 211

Rouleau, F., \& Martin, P. G. 1991, ApJ, 377, 526

Tsuji, T., Aoki, W., \& Ohnaka, K. 1999, in Proc. The Universe as seen by ISO, ed. P. Cox, \& M. F. Kessler, ESA-SP 427, 1, 229

van Leer, B. 1977, J. Comput. Phys., 23, 276

Winters, J. M., Le Bertre, T., Jeong, K. S., Helling, Ch., \& Sedlmayr, E. $2000, A \& A, 361,641$

Winters, J. M., Fleischer, A. J., Le Bertre, T., \& Sedlmayr, E. 1997, A\&A, 326, 305 